\title{
Tendência da mortalidade por doença de Chagas na Bahia: Entre os anos de 2008 a
}

\section{8}

\author{
Trend of mortality due to Chagas disease in Bahia: Between the years 2008 to 2018 \\ Tendencia de mortalidad por enfermedad de Chagas en Bahia: Entre los años 2008 a 2018
}

Recebido: 05/04/2021 | Revisado: 15/04/2021 | Aceito: 24/04/2021 | Publicado: 09/05/2021

Débora Silva Amorim

ORCID: https://orcid.org/0000-0003-0894-9635 Unidade de Ensino Superior de Feira de Santana, Brasil E-mail: amorimdebora_@outlook.com

Misael Silva Ferreira Costa

ORCID: https://orcid.org/0000-0001-8042-2489 Unidade de Ensino Superior de Feira de Santana, Brasil E-mail: ffcost@hotmail.com

\begin{abstract}
Resumo
Introdução: A Doença de Chagas é uma patologia parasitária resultante da infecção pelo protozoário Trypanosoma cruzi, a doença é principalmente transmitida pela forma vetorial, sendo o inseto Triatominae, comumente chamado de barbeiro seu principal vetor. Estima-se que mais de 6 milhões de pessoas estejam infectadas com o Trypanosoma cruzi em todo mundo. Objetivo: O presente artigo teve como objetivo avaliar a tendência de mortalidade por Doença de Chagas na Bahia entre os anos de 2008 a 2018, enfatizando assim, a correlação da doença com as variáveis: sexo, faixa etária e escolaridade. Metodologia: Trata-se de um estudo ecológico, de caráter retrospectivo, onde utilizou-se dados coletados do Sistema de Informação de Mortalidade (SIM) do Departamento de Informática do Sistema Único de Saúde (DATASUS) na seção de informações de saúde do Tabulador Genérico do Domínio Público (TABNET). Resultados e Discussão: Observam-se valores decrescentes para os níveis de mortalidade no Brasil por Doença de Chagas. Entretanto, na Região Nordeste foram registradas 11.309 mortes, desta 6.856 ocorreram no Estado da Bahia, o que é considerado um fator preocupante já que a Região Nordeste possui 9 estados e, apenas a Bahia apresentou mais de $50 \%$ dos óbitos por Doença de Chagas que sucederam nessa região. Conclusão: Pela observação dos dados analisados percebe-se que a Bahia possui uma elevada tendência de mortalidade por Doença de Chagas, fato que está correlacionado com o sexo, faixa etária e escolaridade dos indivíduos que foram a óbitos por essa doença.
\end{abstract}

Palavras-chave: Doença de Chagas; Mortalidade; Saúde pública; Trypanosoma cruzi.

\begin{abstract}
Introduction: Chagas disease is a parasitic pathology resulting from infection by the protozoan Trypanosoma cruzi, the disease is mainly transmitted by the vector form, with the insect Triatominae, commonly called the barber its main vector. It is estimated that more than 6 million people are infected with Trypanosoma cruzi worldwide. Objective: This article aimed to assess the trend of mortality from Chagas Disease in Bahia between the years 2008 to 2018 , thus emphasizing the correlation of the disease with the variables: sex, age and education. Methodology: This is a retrospective ecological study, using data collected from the Mortality Information System (SIM) of the Informatics Department of the Unified Health System (DATASUS) in the health information section of the Generic Domain Tabulator. Public (TABNET). Results and Discussion: Decreasing values are observed for mortality levels in Brazil due to Chagas disease. However, in the Northeast Region 11,309 deaths were registered, of which 6,856 occurred in the State of Bahia, which is considered a worrying factor since the Northeast Region has 9 states and only Bahia had more than $50 \%$ of deaths from Chagas Disease that succeeded in that region. Conclusion: By observing the analyzed data, it can be seen that Bahia has a high mortality trend due to Chagas disease, a fact that is correlated with the sex, age group and education of individuals who died from this disease.
\end{abstract}

Keywords: Chagas Disease; Mortality; Public health; Trypanosoma cruzi.

\section{Resumen}

Introducción: La enfermedad de Chagas es una patología parasitaria resultante de la infección por el protozoo Trypanosoma cruzi, la enfermedad se transmite principalmente por la forma vector, siendo el insecto Triatominae, comúnmente llamado barbero su principal vector. Se estima que más de 6 millones de personas están infectadas con Trypanosoma cruzi en todo el mundo. Objetivo: Este artículo tuvo como objetivo evaluar la tendencia de la mortalidad por Enfermedad de Chagas en Bahía entre los años 2008 a 2018, enfatizando así la correlación de la enfermedad con las variables: sexo, edad y educación. Metodología: Se trata de un estudio ecológico retrospectivo, utilizando datos recopilados del Sistema de Información de Mortalidad (SIM) del Departamento de Informática del 
Sistema Único de Salud (DATASUS) en la sección de información de salud del Tabulador de Dominio Genérico. Público (TABNET). Resultados y discusión: Se observan valores decrecientes para los niveles de mortalidad en Brasil debido a la enfermedad de Chagas. Sin embargo, en la Región Nordeste se registraron 11.309 defunciones, de las cuales 6.856 ocurrieron en el Estado de Bahía, lo que se considera un factor preocupante ya que la Región Nordeste tiene 9 estados y solo Bahía tuvo más del 50\% de las defunciones por Enfermedad de Chagas que triunfó en esa región. Conclusión: Al observar los datos analizados, se puede apreciar que Bahía tiene una alta tendencia de mortalidad por enfermedad de Chagas, hecho que se correlaciona con el sexo, grupo de edad y educación de los individuos que fallecieron por esta enfermedad.

Palabras clave: La Enfermedad de Chagas; Mortalidad; Salud pública; Trypanosoma cruzi.

\section{Introdução}

A Doença de Chagas é uma importante patologia parasitária resultante da infecção pelo protozoário Trypanosoma cruzi, a doença é principalmente transmitida pela forma vetorial, sendo o inseto Triatominae, comumente chamado de barbeiro seu principal vetor, sendo menos frequentemente transmitida, pela ingestão de alimentos contaminados com insetos infectados ou com suas fezes, a transmissão também pode ocorrer por via transplacentária, por transfusão de sangue ou por transplante de órgão (Porfírio et al., 2020).

O Trypanosoma cruzi é o agente etiológico da Tripanosomiase Americana ou Doença de Chagas, o mesmo é um protozoário flagelado que pertence à ordem Kinetoplastida, da família Trypanosomatidae (Galvão, 2014). Estima-se que mais de 6 milhões de pessoas estejam infectadas com o Trypanosoma cruzi em todo mundo. A doença é considerada um problema de saúde pública, estando presente em áreas endêmicas de 21 países da Região das Américas, sendo considerada uma patologia negligenciada que acomete, em especial, populações de renda baixa (Salud, 2020).

As manifestações clínicas decorrentes da infecção por Trypanosoma cruzi podem se manifestar na fase aguda da doença ou pode se arrastar para um quadro crônico, onde o paciente apresentará complicações relevantes em determinados órgãos (Porfírio et al., 2020). Geralmente os sintomas são: febre prolongada, cefaleia, manchas na pele, edema de face ou de membros, aumento do fígado ou baço, cardiopatia aguda, entre outros (Rodrigues et al., 2016). A fase crônica possui grande relevância por ser a forma clínica de maior prevalência na Doença de Chagas (Dias et al., 2016), em alguns casos, a fase crônica é caracterizada pela ausência de sintomas que posteriormente pode evoluir complicações digestiva, cardíaca ou mista. A fase crônica da DC é inicialmente marcada pela presença de uma miocardite fibrosa crônica, de baixa intensidade e incessante, a qual produz dano progressivo ao miocárdio e resulta tardiamente em uma cardiomiopatia chagásica crônica (Simões et al., 2018; Ortiz et al., 2019).

O diagnóstico da doença é feito através de exames laboratoriais, sendo estes o exame parasitológico e o exame sorológico (Rodrigues et al., 2016). O exame parasitológico é essencial na fase aguda da patologia, pois é nesse período que a parasitemia está elevada, tornando-se possível a visualização das formas tripomastigostas do Trypanosoma cruzi nas amostras de fezes. Entretanto, após 12 semanas da infecção, os níveis de parasitemia decaem, transformando-se indetectáveis em microscopia (Porfírio et al., 2020). Posteriormente a doença pode evoluir para fase crônica sendo possível ser diagnosticada através de xenodiagnóstico, hemocultura ou PCR (Rodrigues et al., 2016). Todavia, o diagnóstico da Doença de Chagas na fase crônica, segundo o II Consenso Brasileiro em Doença de Chagas (2015), é realizado essencialmente, por exames sorológicos, podendo ser utilizados as técnicas: ELISA, hemaglutinação e imunofluorescência. Hemocultura, xenodiagnóstico ou qualquer outro critério parasitológico possui baixa sensibilidade na fase crônica, portanto, não devem ser utilizados sozinho como meio diagnóstico (Dias et al., 2016).

Em 2006, a Organização Mundial de Saúde (OMS) presenteou o Brasil com o certificado de erradicação da transmissão da doença pela forma vetorial, o que representou a erradicação do vetor, o inseto Triatominae e da doença, mas ainda há muitos outros vetores da doença como os insetos Hemiptera e Reduviidae. (Dias et al., 2016; Simões et al., 2018). Entretanto, é sabido que a transmissão da doença continua acontecendo por meio de surtos mediados por outros mecanismos de transmissão. 
Diante da grande incidência de casos da Doença de Chagas no Brasil, principalmente no estado da Bahia, faz-se necessário a averiguação da tendência de mortalidade decorrente dessa patologia. O presente artigo teve como objetivo avaliar a tendência de mortalidade por Doença de Chagas na Bahia entre os anos de 2008 a 2018, enfatizando assim, a correlação da doença com as variáveis: sexo, faixa etária e escolaridade.

\section{Metodologia}

Trata-se de um estudo ecológico, de caráter retrospectivo, onde utilizou-se dados coletados do Sistema de Informação de Mortalidade (SIM) do Departamento de Informática do Sistema Único de Saúde (DATASUS) na seção de informações de saúde do Tabulador Genérico do Domínio Público (TABNET). Para realizar as buscas dos dados, utilizou-se a $10^{\mathrm{a}}$ revisão da versão brasileira da Classificação Internacional de Doenças (CID-BR-10), para Doenças de Chagas.

Foram analisados dados da tendência da mortalidade por Doença de Chagas no Estado da Bahia entre os anos de 2008 a 2018, analisou-se as variáveis: faixa etária, sexo e escolaridade. Analisou-se também a tendência de mortalidade no Brasil e na Região Nordeste no período proposto pelo presente trabalho, para possibilitar então a comparação de dados dos óbitos entre o Brasil, a Região Nordeste e a Bahia.

Realizou-se uma pesquisa bibliográfica nas bases de dados eletrônicas Scientific Eletronic Library Online (SciELO) e Biblioteca Virtual em Saúde (BVS), utilizando-se de palavras chaves os termos: "Doença de Chagas", "Trypanosoma cruzi" e "Doença de Chagas AND Brasil". As informações adquiridas sobre a Doença de Chagas fornecidas pelas ferramentas do Ministério da Saúde foram analisadas, utilizando-se o programa Microsoft Word Excel 2016 ®. Utilizaram-se os dados fornecidos por meio de tabelas e gráficos de acordo com o objetivo do trabalho.

\section{Resultados e Discussão}

No período analisado, observam-se valores decrescentes para os níveis de mortalidade no Brasil por Doença de Chagas, nota-se que o Brasil apresentou uma tendência de 50.959 óbitos decorrentes dessa patologia. Entretanto, na Região Nordeste foram registradas 11.309 mortes, desta 6.856 ocorreram no Estado da Bahia, o que é considerado um fator preocupante já que a Região Nordeste possui 9 estados e, apenas a Bahia apresentou mais de 50\% dos óbitos por Doença de Chagas que sucederam nessa região (Tabela 1).

Tabela 1 - Comparativa da tendência de mortalidade por Doença de Chagas no Brasil, Nordeste e Bahia entre 2008 a 2018.

\begin{tabular}{lccc}
\hline \multicolumn{4}{c}{ Comparativa da tendência de mortalidade por doença de Chagas } \\
\hline ANO & BRASIL & NORDESTE & BAHIA \\
$\mathbf{2 0 0 8}$ & 5.096 & 1.077 & 646 \\
$\mathbf{2 0 0 9}$ & 4.741 & 1.010 & 609 \\
$\mathbf{2 0 1 0}$ & 4.876 & 1.099 & 682 \\
$\mathbf{2 0 1 1}$ & 4.673 & 1.083 & 661 \\
$\mathbf{2 0 1 2}$ & 4.650 & 1.070 & 660 \\
$\mathbf{2 0 1 3}$ & 4.628 & 1.015 & 628 \\
$\mathbf{2 0 1 4}$ & 4.428 & 988 & 603 \\
$\mathbf{2 0 1 5}$ & 4.472 & 955 & 578 \\
$\mathbf{2 0 1 6}$ & 4.461 & 1.006 & 573 \\
$\mathbf{2 0 1 7}$ & 4.543 & 984 & 603 \\
$\mathbf{2 0 1 8}$ & 4.391 & 1.022 & 613 \\
\hline
\end{tabular}

Fonte: Autores; Dados do SIM/DATASUS (2021). 
Entre os anos de 2008 a 2018 foram registrados 6.856 óbitos por Doença de Chagas no Estado da Bahia. Segundo os dados encontrados no Sistema de Informação de Mortalidade (SIM) do Departamento de Informática do Sistema Único de Saúde (DATASUS), houve 646 óbitos em 2008, já em 2009 ocorreram 609 mortes, entretanto, em 2010 foram registados 682 óbitos, em contrapartida aconteceram 661 mortes em 2011, já em 2012 houve uma tendência de 660 óbitos, com tudo, em 2013 aconteceram 620 mortes, todavia ocorreram 603 mortes em 2014, no que se refere a 2015 foram registrados 578 óbitos, em contrapartida, ocorreram 573 mortes em 2016, já em 2017 sucederam 603 óbitos, entretanto, em 2018 aconteceram 613 óbitos (Figura 1).

Figura 1 - Taxa de Mortalidade por Doença de Chagas entre os anos de 2008 a 2018.

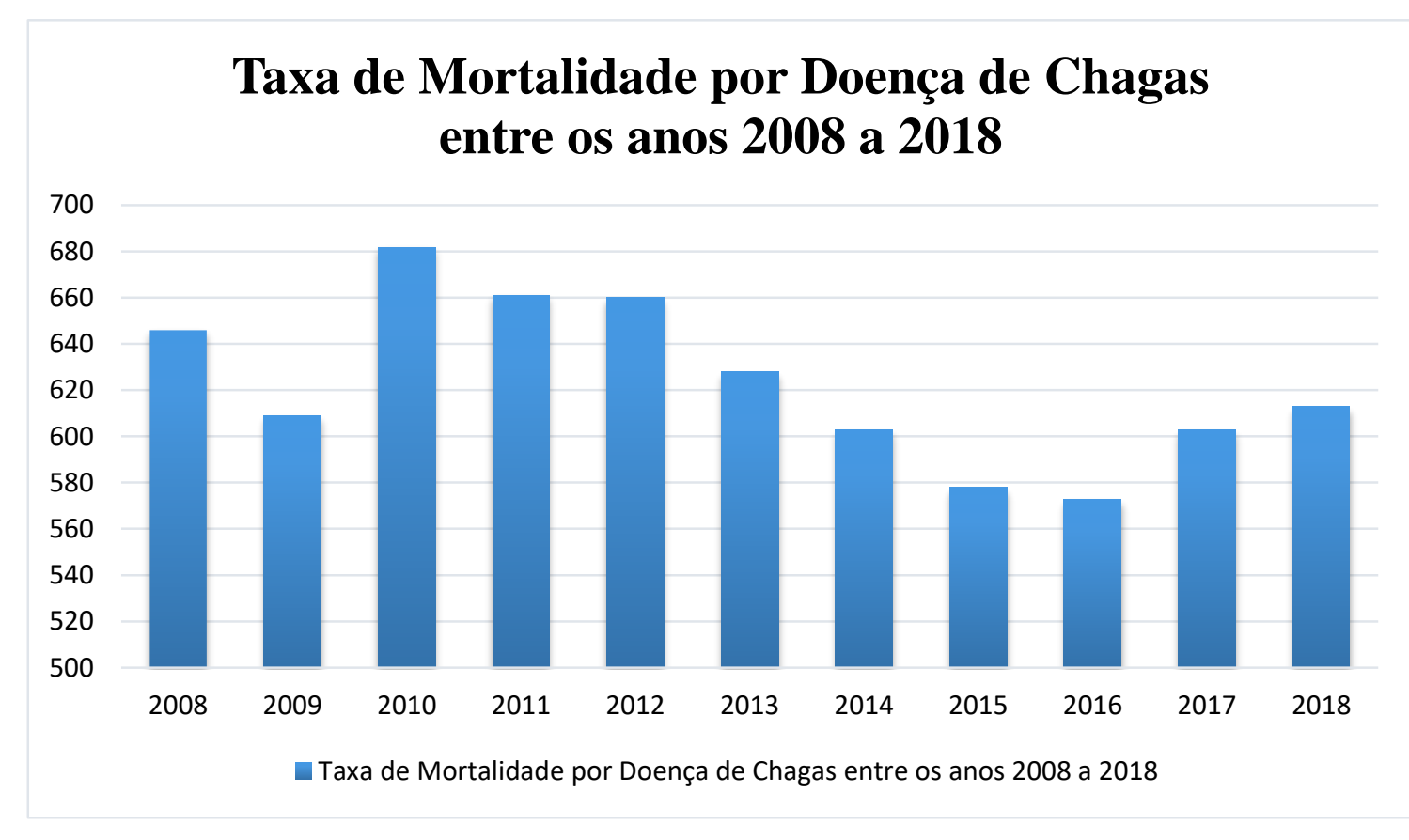

Fonte: Autores; Dados do SIM/DATASUS (2021).

Em relação aos óbitos por faixa etária, observou-se os dados da tendência de mortalidade por Doenças de Chagas nas seguintes faixas etárias: de 30 a 39 anos, 40 a 49 anos e 50 a 59 anos. Levando em consideração os dados obtidos no SIM/DATASUS, a faixa etária que demostrou uma maior tendência de óbitos entre os anos de 2008 a 2018 foi a faixa etária de 50 a 59 anos com 1.356, percebe-se que a faixa etária é um fator de importância, pois pessoas com uma maior idade são mais propensas a vim a óbito devido essa doença (Tabela 1). 
Tabela 2 - Óbitos por faixa etária nos anos de 2008 a 2018.

\begin{tabular}{cccc}
\hline \multicolumn{5}{c}{ Óbitos por faixa etária } \\
\hline ANO & 30 A 39 ANOS & 40 A 49 ANOS & 50 A 59 ANOS \\
$\mathbf{2 0 0 8}$ & 34 & 85 & 167 \\
$\mathbf{2 0 0 9}$ & 37 & 78 & 130 \\
$\mathbf{2 0 1 0}$ & 43 & 65 & 156 \\
$\mathbf{2 0 1 1}$ & 33 & 87 & 122 \\
$\mathbf{2 0 1 2}$ & 29 & 75 & 125 \\
$\mathbf{2 0 1 3}$ & 30 & 66 & 138 \\
$\mathbf{2 0 1 4}$ & 20 & 52 & 108 \\
$\mathbf{2 0 1 5}$ & 26 & 49 & 91 \\
$\mathbf{2 0 1 6}$ & 24 & 54 & 92 \\
$\mathbf{2 0 1 7}$ & 15 & 49 & 116 \\
$\mathbf{2 0 1 8}$ & 12 & 49 & 111 \\
\hline
\end{tabular}

Fonte: Autores; Dados do SIM/DATASUS (2021).

No que se refere ao sexo analisaram-se os dados presente no SIM/DATASUS, dos óbitos no sexo masculino e feminino entre os anos de 2008 a 2018 no estado da Bahia. Constataram-se que o sexo masculino possui uma maior predisposição de mortalidade devido a Doenças de Chagas, pois no período de 2008 a 2018 aconteceram 4.127 óbitos devido a presente doença nos homens, entretanto o sexo feminino apresentou apenas 2.729 óbitos decorrentes dessa doença (Figura 2).

Figura 2 - Comparativa da tendência de mortalidade em relação ao sexo.

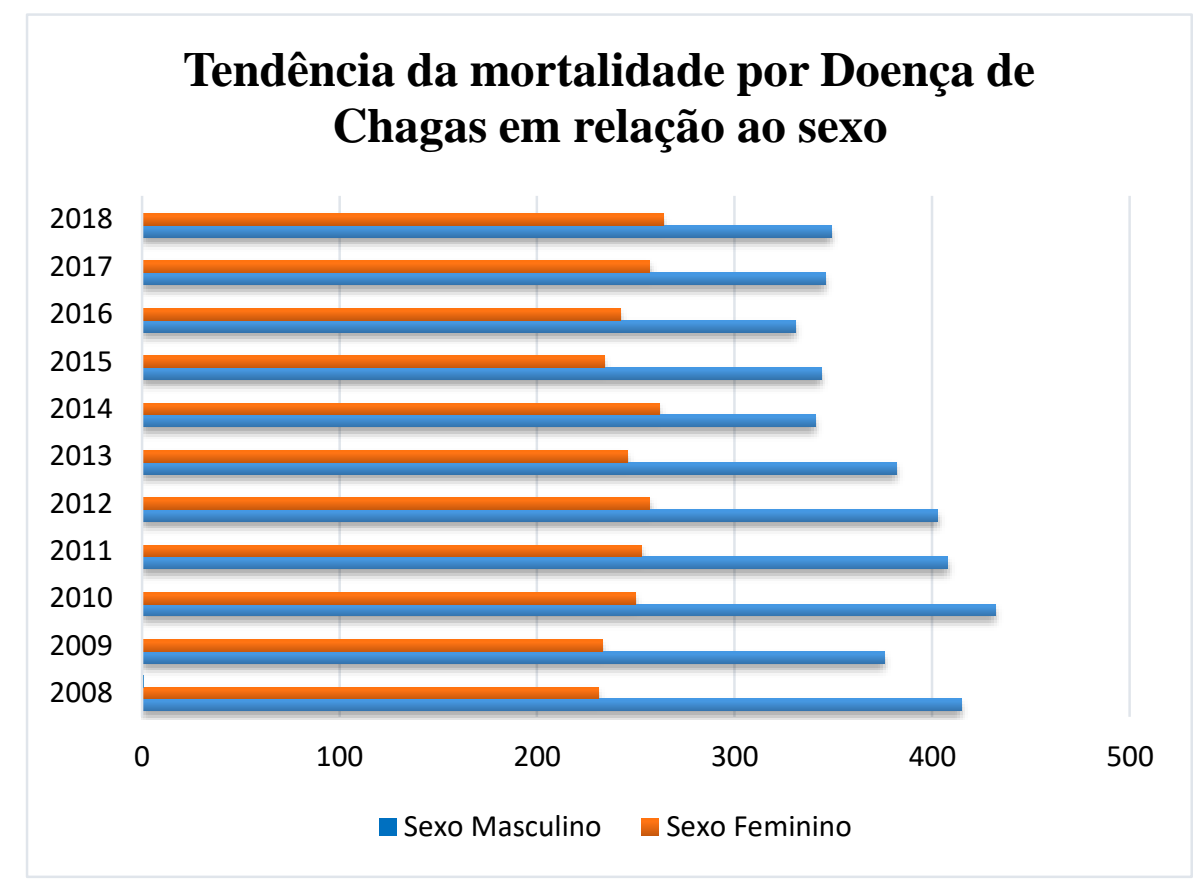

Fonte: Autores; Dados do SIM/DATASUS (2021).

Os óbitos ocorridos na Bahia por Doença de Chagas entre 2008 a 2018, também estão correlacionados com o grau de escolaridade da população desse estado, visto que os dados obtidos demostraram que os indivíduos que apresentaram nenhum e/ou baixo grau de escolaridade apresentaram uma maior tendência de mortalidade, em contrapartida, os indivíduos com grau médio e/ou alto de escolaridade possuíram uma menor tendência de mortalidade (Figura 3). 
Figura 3 - Relação do grau de escolaridade com a tendência de mortalidade por Doença de Chagas.

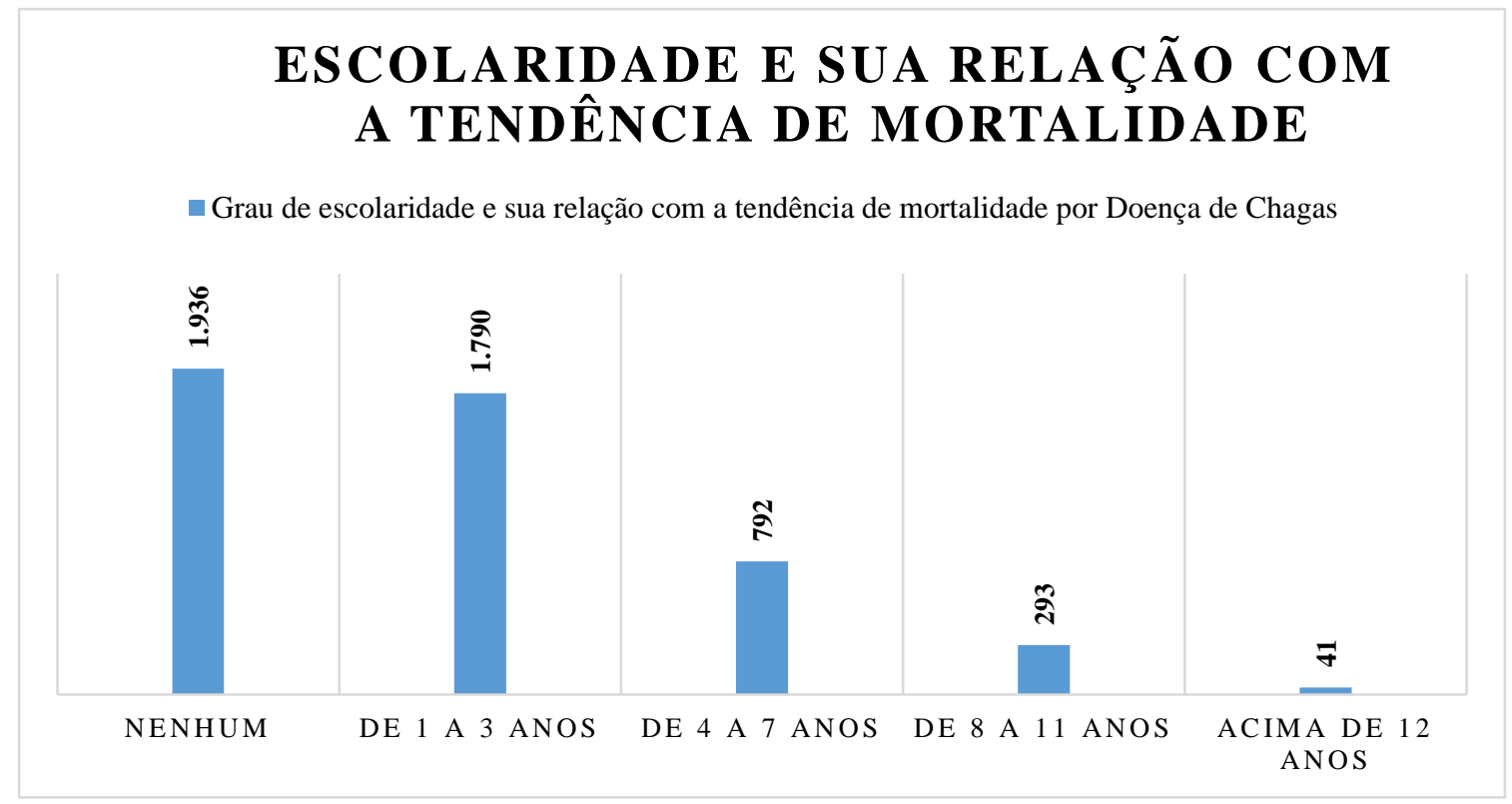

Fonte: Autores; Dados do SIM/DATASUS (2021).

O padrão de mortalidade por Doença de Chagas resulta da intensidade da infecção, da região geográfica, da linhagem do parasito e da condição da imunidade do hospedeiro. No período estudado, observaram-se uma diminuição dos óbitos totais, o que se dá pela erradicação das formas de transmissões vetorial e transfusional do Trypanosoma cruzi. Entretanto, ao analisarse os óbitos ocorridos, notou-se que houve uma maior mortalidade entre os homens do que entre as mulheres, todavia não se sabe ainda se o sexo pode ser considerado um fator de risco na Doença de Chagas (Mota et al., 2014).

O acometimento cardíaco da doença, na fase crônica, aumenta a morbidade e mortalidade, além de estar diretamente relacionada com a maior ocorrência de acidentes vascular encefálicos, outro fator de risco que pode levar à morte. Desta forma, a insuficiência cardíaca, eventos tromboembólicos e morte súbita são os principais preditores de morte na Doença de Chagas crônica. Os indicadores de idade ao morrer refletiram características da cronicidade e o impacto de longo prazo das medidas para o controle e tratamento da doença (Simões et al., 2018). Desses indicadores a maior proporção de óbitos aconteceram acima de 30 a 39 anos de idade, o deslocamento das mortes para grupos etários de 40 anos ou mais e os coeficientes mais elevados foram em pessoas com 50 a 59 anos, o que aponta a maior sobrevida dos doentes portadores do Trypanosoma cruzi (Santo, 2009).

Mediante os dados apresentados no presente estudo, percebe-se que embora tenha ocorrido a erradicação das transmissões vetoriais e transfusionais, estimativas matemáticas mostram que os óbitos por Doença de Chagas ainda continuaram sendo um obstáculo da saúde pública durante décadas (Nóbrega et al., 2014), seja devido à transmissão oral por consumo de alimentos contendo triatomíneos ou suas dejeções, seja por mudanças climáticas e ambientais acontecidas na região. Entre os alimentos possivelmente responsáveis pela transmissão da doença, os principais são: açaí, caldo de cana, alimentos não devidamente cozidos e carne de caça semicrua. No tocante às mudanças ambientais favorecedoras do surgimento do triatomíneo no estado da Bahia, são citados, como fatores predisponentes, o desmatamento, a condições de vida, o crescimento desordenado das cidades, e as mudanças climáticas provenientes do estado (Santos et al., 2018). 


\section{Conclusão}

A Doença de Chagas é considerada uma patologia negligenciada que afeta na grande maioria dos casos indivíduos de baixa venda, mesmo diante da certificação de interrupção de transmissão da Doença de Chagas pelo vetor em 2006 , nota-se que vários aspectos não foram levados em consideração, visto que na Região Nordeste, principalmente, na Bahia grande incidência de casos e números elevados de óbitos. Pela observação dos dados analisados percebe-se que a Bahia possui uma elevada tendência de mortalidade por Doença de Chagas, situação que está correlacionado com o sexo, faixa etária e escolaridade dos indivíduos que foram a óbitos por essa doença.

O Brasil representa uma das principais áreas endêmicas dessa doença no mundo, circunstância que define a Doença de Chagas como um problema de Saúde Pública, devido sua grande complexidade para prevenção e controle. Diante do crescente números de mortes ocasionadas por essa patologia, é notória a necessidade de medidas de controle do barbeiro, para impedir a proliferação da doença, bem como, ações de promoção e prevenção, atenção à saúde para diagnóstico e tratamento oportunos.

\section{Referências}

Brasil. SIM/DATASUS (2020). Óbitos por Doença de Chagas. http://tabnet.datasus.gov.br/cgi/tabcgi.exe?sim/cnv/obt10uf.def.

Cruz, C. A. B., et al. (2020). Tecnologias que empregam fármacos antiparasitários para tratamento da doença de Chagas. Rev. Eletron. Comum. Inf. Inov. Saúde. 10(1).

Dias, J. C. P. et al. (2016). II Consenso Brasileiro em Doença de Chagas, Epidemol. Serv. Saúde.

Galvão, C. (2014). Vetores da doença de Chagas no Brasil. Sociedade Brasileira de Zoologia.

Guariento, M. E. et al. (2011). Perfil Clínico de Idosos Portadores de Doença de Chagas Atendidos em serviço de Referência. Rev. Soc. Bras. Med. 9(1).

Lima, N. de J. de F.; Farias, M. P. O. (2020). Estudo retrospectivo e transversal dos casos de doença de Chagas aguda no Brasil de 2007 a 2017 . PUBVET. $14(10), 1-9$

Mota, J. C. da et al. (2014). Estimativa de Taxa de Mortalidae e Taxa de Incidência de Sequelas Cardíacas e Digestivas por Doença de Chagas no Brasil, 2008. Epidemiol. Serv. Saúde. 234, 711-720.

Ortiz, J. V. et al. (2019). Avaliação Cardíaca na Fase da Doença de Chagas com Evolução Pós-Tratamento em Pacientes Atendidos no Estado do Amazonas, Brasil. Arq. Bras. Cadiol. 112(3).

Porfírio, D. M., et al. (2020). Prevalência de Doença de Chagas em Idosos no Estado do Pará: Uma Análise Retrospectiva. Brazilian Jouenal of health Review. 3(4), 9142-9152.

Rodrigues, R. P. de S., et al. (2016). Características epidemiologias, zoonóticas, clínicas, patológicas e diagnósticos da doença de Chagas. PUBVET. 10(3), 200-206.

Salud, O. P. de la (2020). Síntesis de evidencia: Guía para el diagnóstico y el tratamento de la enfermidade de Chagas. Rev. Panam. Salud. Publica 44.

Santo, A. H. (2009). Tendência da Mortalidade Relacionada à Doença de Chagas, Estado de São Paulo, Brasil, 1985 a 2006: Estudo Usando Causas Múltiplas de Morte. Rev. Panam. Salud Pública. 26(4).

Santos, C. B. et al. (2018). Perfil dos Óbitos por Doença de Chagas no Nordeste do Brasil, Entre os Anos de 2005 e 2014 . Convención Internacional de Salud. Cuba Salud.

Simões, M. V. et al. (2018). Cardiomiopatia da Doença de Chagas. International Journal of Cardiovascular Sciences. 31(2).

Souza, C. N. P., de et al. (2014). Fatores Contribuintes à Ocorrência de Mortalidade por Doença de Chagas. Rev. Bras. Biom. 32(4), 544-552.

Vargas, A., et al. (2018). Investigação de surto de doença de Chagas aguda na região extra-amazônica, Rio Grande do Norte, Brasil, 2016. Cad. Saúde Pública. 34(1). https://doi.org/10.1590/0102-311X00006517. 